\title{
ON THE PROXIMATE LINEAR ORDERS OF ENTIRE DIRICHLET SERIES $\left({ }^{(}\right)$
}

BY

\author{
A. G. AZPEITIA
}

1. Let $f(s)(s=\sigma+i t)$ be an entire function defined by a Dirichlet series (1)

$$
\sum_{n=0}^{\infty} a_{n} e^{\lambda_{n} s}, \quad 0=\lambda_{0}<\lambda_{1}<\cdots<\lambda_{n} \uparrow \infty,
$$

absolutely convergent for all $s$.

The linear order, or order $(R)$, and the lower linear order of $f(s)$ are defined, [4, p. 77] and [3, p. 96], as the numbers $\rho=\lim \sup _{\sigma=\infty} \log \log M(\sigma) / \sigma$ $(0 \leqq \rho \leqq \infty)$ and

$$
\tau=\liminf _{\sigma=\infty} \log \log M(\sigma) / \sigma \quad(0 \leqq \tau \leqq \infty)
$$

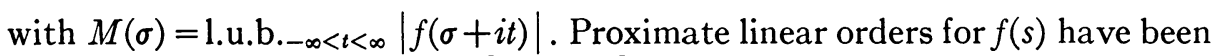
defined by Sunyer i Balaguer [7, p. 28] by extending in the natural way the notion of Lindelöf's proximate order [6, p. 326].

Since $\log M(\sigma)$ is an increasing convex [4, pp. 74-75] and therefore continuous [8, p. 172] function of $\sigma$, the arguments of Shah [5] and this author [2, pp. 20-25] can be applied, with trivial modifications, to the present case.

The following propositions of existence of linear proximate orders $R(\sigma)$ and linear lower proximate orders $T(\sigma)$, are thus easily obtained:

(A) If $0<\rho<\infty$, then for any given number $a(0<a<\infty)$, there exists a positive continuous function $R(\sigma)$ such that: (i) the derivatives $R^{\prime}(\sigma)$ and $R^{\prime \prime}(\sigma)$ exist everywhere but for isolated points where $R^{\prime}(\sigma \pm 0)$ and $R^{\prime \prime}(\sigma \pm 0)$ exist. (ii) $\lim _{\sigma=\infty} \sigma R^{\prime}(\sigma)=\lim _{\sigma=\infty} \sigma R^{\prime \prime}(\sigma)=0$. (iii) $\lim _{\sigma=\infty} R(\sigma)=\rho$. (iv) lim $\sup _{\sigma=\infty} \log M(\sigma) / \exp [\sigma R(\sigma)]=a$.

(B) If $0<\tau<\infty$, then for any given number $b(0<b<\infty)$, there exists $a$ continuous positive function $T(\sigma)$ satisfying conditions (i) and (ii) of part (A) and such that: (iii') $\lim _{\sigma=\infty} T(\sigma)=\tau$. (iv') $\lim \inf _{\sigma=\infty} \log M(\sigma) / \exp [\sigma T(\sigma)]=b$.

In what follows we will study the upper and lower limits for $\sigma \rightarrow \infty$ of the quotient $\log m(\sigma) / \exp [\sigma P(\sigma)]$ where $m(\sigma)$ is defined as

$$
m(\sigma)=\max _{n \geqq 0}\left|a_{n} \exp \left[\lambda_{n}(\sigma+i t)\right]\right|, \quad(n=0,1,2 \cdots),
$$

and $P(\sigma)$ is a function having first and second derivatives for all $\sigma$ and such

Received by the editors September 18, 1961.

(1) This work is part of a research supported by the National Foundation under grant NSF G14142. 
that: $(\alpha) \lim _{\sigma=\infty} \sigma P^{\prime}(\sigma)=\lim _{\sigma=\infty} \sigma P^{\prime \prime}(\sigma)=0$. ( $\left.\beta\right) 0<p=\lim _{\sigma=\infty} P(\sigma)<\infty$. (For notations and terminology see also [1].)

All our conclusions will be valid if $M(\sigma)$ is substituted for $m(\sigma)$ provided the asymptotic equivalence $\lim _{\sigma=\infty} \log M(\sigma) / \log m(\sigma)=1$ holds. Sufficient conditions to guarantee this equivalence are given in [7, Theorem 5] and [1, Theorem 2].

2. If $x=\exp [\sigma P(\sigma)]$, then the assumptions made on $P(\sigma)$ imply that the inverse function $\sigma=\phi(x)$ exists, as well as $\phi^{\prime}(x)$ and $\phi^{\prime \prime}(x)$, for all positive large enough $x$, and has the following properties that will be used later.

With $\varphi(x)$ defined by the condition $\phi(x)=\log x / \varphi(x)$ we have

$$
\begin{aligned}
\lim _{x=\infty} \varphi(x) & =p, \\
\sigma P^{\prime}(\sigma) & =x \varphi(x) \varphi^{\prime}(x) \log x /\left[\varphi(x)-x \varphi^{\prime}(x) \log x\right], \\
x \varphi^{\prime}(x) \log x & =\sigma P(\sigma) P^{\prime}(\sigma) /\left[P(\sigma)+\sigma P^{\prime}(\sigma)\right] .
\end{aligned}
$$

Since $x \rightarrow+\infty$ if and only if $\sigma \rightarrow+\infty$ it follows that

$$
\lim _{\sigma+\infty} \sigma P^{\prime}(\sigma)=\lim _{x=\infty} x \varphi^{\prime}(x) \log x=0 .
$$

From (2) and the definition of $\varphi(x)$ we obtain

$$
P^{\prime}(\sigma)=x \varphi^{\prime}(x) \varphi(x)^{2} /\left[\varphi(x)-x \varphi^{\prime}(x) \log x\right]
$$

and by differentiation and using (3), it is easily seen that for corresponding values of $\sigma$ and $x$ approaching $+\infty$ we have $\sigma P^{\prime \prime}(\sigma) \sim\left[x^{2} \varphi^{\prime \prime}(x) \log x\right] / \varphi(x)$ and therefore

$$
\lim _{x=\infty} x^{2} \varphi(x) \log x=0
$$

3. We consider next, the properties of the function

$$
\Gamma(\lambda, \alpha)=\exp [(\lambda / p) \log \alpha-\lambda \log \lambda / \varphi(\lambda)]
$$

where $\lambda$ is a real positive variable and $\alpha$ a real positive parameter. We also consider the related function of two real variables $\lambda, \sigma$ given by $\Gamma(\lambda, \alpha) \exp (\sigma \lambda)$ $(0 \leqq \lambda<\infty,-\infty<\sigma<\infty)$. The function

$$
y=-\log \Gamma(\lambda, \alpha)
$$

is convex for all large values of $\lambda$ since by differentiating with respect to $\lambda$, and writing $\varphi$ for $\varphi(\lambda)$ we obtain

$$
y^{\prime \prime}=\left[1 /\left(\lambda \varphi^{3}\right)\right]\left[\varphi^{2}-\varphi \lambda^{2} \varphi^{\prime \prime} \log \lambda-2 \varphi \lambda \varphi^{\prime} \log (\lambda e)+2\left(\lambda \varphi^{\prime} \log \lambda\right)^{2} / \lambda\right]>0
$$

by virtue of (2), (3) and (4), for all large enough $\lambda$. In view of the facts that $y \uparrow \infty, y^{\prime} \uparrow \infty, y^{\prime \prime}>0$ we can conclude that the function of $\sigma$ 


$$
\mu(\sigma, \alpha)=\max _{\lambda \geqq 0} \Gamma(\lambda, \alpha) \exp (\sigma \lambda)
$$

does exist. The argument is similar to the standard one used in the case of a Newton-Hadamard polygon [1, pp. 717-718; 6, p. 274] and the strict convexity of (5) implies that for each large $\sigma$ the maximum is obtained for the value of $\lambda=\lambda(\sigma, \alpha)$ uniquely defined by the condition that the slope of the curve (5) be equal to $\sigma$, that is to say

$$
\sigma=-(1 / p) \log \alpha+(1 / \varphi) \log (\lambda e)-\left(1 / \varphi^{2}\right)\left(\lambda \varphi^{\prime} \log \lambda\right) .
$$

Substituting in (6), using the definition of $\Gamma(\lambda, \alpha)$ and writing $\lambda$ for $\lambda(\sigma, \alpha)$, we obtain

$$
\log \mu(\sigma, \alpha)=(\lambda / \varphi)\left[1-(1 / \varphi)\left(\lambda \varphi^{\prime} \log \lambda\right)\right] \sim \lambda / p .
$$

On the other hand, the assumption ( $\alpha$ ) implies that for any $\alpha=\alpha(\sigma)$ bounded in absolute value

$$
P(\sigma+\alpha(\sigma))=P(\sigma)+o(1 / \sigma)
$$

and consequently from (7) and (9) we deduce $\sigma P(\sigma)=[-(1 / p) \log \alpha+(1 / \varphi) \log (\lambda e)+O(1 / \lambda)][P(\log \lambda / \varphi)+o(\varphi / \log \lambda)]$, and since by the definition of $\varphi(\lambda)$ we know that $P(\log \lambda / \varphi) \equiv \varphi(\lambda)$ we can conclude that $\sigma P(\sigma)=-\log \alpha+\log (\lambda e)+O(1 / \lambda)$, which together with (8) implies

$$
\lim _{\sigma=\infty} \log \mu(\sigma, \alpha) / \exp [\sigma P(\sigma)]=\alpha /(p e)
$$

and, again by (8)

$$
\lim _{\sigma=\infty} \lambda(\sigma, \alpha) / \exp [\sigma P(\sigma)]=\alpha / e .
$$

4. We denote by $\pi$ the Newton-Hadamard polygon corresponding to the sequence of points with coordinates $\lambda=\lambda_{n}, y=-\log \left|a_{n}\right|$ on the cartesian plane $(\lambda, y)$. (See $[1$, p. $718 ; 6$, p. 274$]$ for terminology and properties related to $\pi$.) The value of $\log m(\sigma)$ is given for each $\sigma$ by the maximum difference between the ordinates of the line $y=\lambda \sigma$ and the polygon $\pi$ and it is achieved for all the corresponding values of $n$. The non-negative integers $n_{i}$ such that $\lambda_{n_{i}}$ are the abscissas of the vertices of $\pi$ are called principal indices and they form a strictly increasing sequence $I \equiv\left\{n_{i}\right\},\left(i=0,1,2, \cdots ; n_{0}=0\right)$, which coincides with the sequence of values taken by the increasing step function $[1$, p. 718] defined by

$$
n(\sigma)=\max _{n \geqq 0}\left\{n|m(\sigma)=| a_{n} \exp \left(\sigma \lambda_{n}\right) \mid\right\} .
$$

For any choice of $P(\sigma)$ as defined in $\S 1$, and any given strictly increasing 
sequence $J \equiv\left\{n_{j}\right\},(j=0,1,2, \cdots)$ of non-negative integers $n_{j}$, we will define the non-negative finite or infinite numbers $A, B, L, Q_{J}, l_{J}$ by the following equalities (in particular for the sequence $I$ we define $Q_{I}$ and $l_{I}$ ):

$$
\begin{aligned}
A & =\limsup _{\sigma=\infty} \log m(\sigma) / \exp [\sigma P(\sigma)], \\
B & =\liminf _{\sigma=\infty} \log m(\sigma) / \exp [\sigma P(\sigma)], \\
(p e L)^{1 / p} & =\limsup _{n=\infty}\left|a_{n}\right|^{1 / \lambda_{n}} \exp \phi\left(\lambda_{n}\right), \\
\left(p e Q_{J}\right)^{1 / p} & =\liminf _{j=\infty}\left|a_{n_{j}}\right|^{1 / \lambda_{n_{j}}} \exp \phi\left(\lambda_{n_{j}}\right), \\
l_{J} & =\limsup _{j=\infty} \lambda_{n_{j+1}} / \lambda_{n_{j}} .
\end{aligned}
$$

Under these definitions and notations we will now apply to the present problem, the technique used by Valiron $[9$, p. 42] in the case of Taylor series, to obtain the following results:

TheOREM 1. Let $P(\sigma)$ be chosen satisfying the conditions stated above. Then, $A=L$.

Proof.Let us assume first that $0<A<\infty$. With $\alpha=A+\epsilon$ it follows from (10) and (13) that for all $\sigma$ large enough

$$
m(\sigma)<\mu[\sigma,(A+\epsilon) p e] .
$$

On the other hand with $\alpha=A-\epsilon$ we obtain

$$
m\left(\sigma_{k}\right)>\mu\left[\sigma_{k},(A-\epsilon) p e\right] \quad \text { for some } \sigma_{k} \uparrow \infty \quad(k=0,1,2, \cdots) .
$$

The inequality (18) implies that from some $n$ on

$$
\left|a_{n}\right|<\Gamma\left[\lambda_{n},(A+\epsilon) p e\right]
$$

since, otherwise there would be a sequence $M \equiv\left\{n_{m}\right\} \quad(m=0,1,2, \cdots)$, such that $\left|a_{n_{m}}\right| \geqq \Gamma\left[\lambda_{n_{m}},(A+\epsilon) p e\right]$ and for those values of $\sigma=\sigma_{m}$ such that $\lambda_{n_{m}}=\lambda\left[\sigma_{m},(A+\epsilon) p e\right]$ we would have, according to the definition of $\lambda(\sigma, \alpha)$ : $m\left(\sigma_{m}\right) \geqq\left|a_{n_{m}} \exp \left(\sigma_{m} \lambda_{n_{m}}\right)\right| \geqq \Gamma\left[\lambda_{n_{m}},(A+\epsilon) p e\right] \exp \left(\sigma_{m} \lambda_{n_{m}}\right)=\mu\left[\sigma_{m},(A+\epsilon) p e\right]$ which contradicts (18). From (19) we conclude that there is a sequence $J \equiv\left\{n_{j}\right\}$ such that

$$
\left|a_{n_{j}}\right|>\Gamma\left[\lambda_{n_{j}},(A-\epsilon) p e\right]
$$

because, otherwise, it would be $\left|a_{n}\right| \leqq \Gamma\left[\lambda_{n},(A-\epsilon) p e\right]$ from some $n$ on and therefore for all large $\sigma, m(\sigma)=\left|a_{n(\sigma)} \exp \left(\lambda_{n(\sigma)} \sigma\right)\right| \leqq \Gamma\left[\lambda_{n(\sigma)},(A-\epsilon) p e\right] \exp \left(\lambda_{n(\sigma)} \sigma\right)$ $\leqq \mu[\sigma,(A-\epsilon) p e]$ contradicting (19).

From the definition of $\Gamma(\lambda, \alpha)$ and (20), (21) and (15) we have $A=L$. If $A=0$ the inequalities (18) and (20) hold for any $\epsilon>0$. It follows that 
$L<\epsilon$ and therefore $L=0=A$. If $A=\infty$ then the inequalities (19) and (21) hold with any arbitrarily large number substituted for $A$ and the conclusion $A=L=\infty$ follows immediately.

TheOREM 2. Let $P(\sigma)$ satisfy the same conditions as above. Then $Q_{I} \geqq B$.

Proof. Assume first $0<B<\infty$. By (14) and (10) we have $m(\sigma)$ $>\mu[\sigma,(B-\epsilon) p e]$ and therefore the polygon $\pi$ is dominated by the curve $y=-\log \Gamma[\lambda,(B-\epsilon) p e]$ for all real values of $\lambda$ and, in particular, $-\log \left|a_{n_{i}}\right|$ $<-\log \Gamma\left[\lambda_{n_{i}},(B-\epsilon) p e\right]$ for the sequence $I$ and the result follows.

If $B=\infty$ all the previous inequalities hold with any arbitrarily large number substituted for $B$ and therefore $Q_{I}=\infty=B$. If $B=0$ the result is trivial.

THEOREM 3. Under the same assumptions for $P(\sigma)$ and with any $J$ such that $l_{J}<\infty$ we have $B \geqq Q_{J} / l_{J}$.

Proof. If $0<Q_{J}<\infty$ then by (16) we have $\left|a_{n_{j}}\right|>\Gamma\left[\lambda_{n_{j}},\left(Q_{J}-\epsilon\right) p e\right]$ for all large $j$.

If the sequence $\sigma_{j} \uparrow \infty$ is defined by $\lambda_{n_{j}}=\lambda\left[\sigma_{j},\left(Q_{J}-\epsilon\right) p e\right]$ then for all $\sigma$ such that $\sigma_{j} \leqq \sigma<\sigma_{j+1}$ we obtain by (10), with $\alpha=\left(Q_{J}-\epsilon\right) p e$

(22) $\log m(\sigma) \geqq \log m\left(\sigma_{j}\right)>\log \mu\left[\sigma_{j},\left(Q_{J}-\epsilon\right) p e\right]=\left(Q_{J}-\epsilon\right) \exp \left[\sigma_{j} P\left(\sigma_{j}\right)\right] u\left(\sigma_{j}\right)$,

where $\lim _{j=\infty} u\left(\sigma_{j}\right)=1$. On the other hand by (11)

$$
\exp [\sigma P(\sigma)] / \exp \left[\sigma_{j} P\left(\sigma_{j}\right)\right] \sim \lambda\left[\sigma,\left(Q_{J}-\epsilon\right) p e\right] / \lambda\left[\sigma_{j},\left(Q_{J}-\epsilon\right) p e\right]=\psi(\sigma),
$$

and since, by (23) and (17), $1 \leqq \psi(\sigma)<\lambda_{n_{j+1}} / \lambda_{n_{j}}<l_{J}+(\epsilon / 2)$ for all large $j$, it follows

$$
1-\epsilon<\exp [\sigma P(\sigma)] / \exp \left[\sigma_{J} P\left(\sigma_{j}\right)\right]<l_{J}+\epsilon
$$

and by $(22), \log m(\sigma) / \exp [\sigma P(\sigma)] \geqq\left(Q_{J}-\epsilon\right) /\left(l_{J}+\epsilon\right)$ for all large $\sigma$ and since $\epsilon$ is arbitrarily small we have finally $B \geqq Q_{J} / l_{J}$. The usual argument shows that the conclusion is equally valid when $Q_{J}=\infty$ and the proof is complete.

Finally for the case of finite order and regular growth, i.e.: $0<\tau=\rho<\infty$, we have under the same assumptions for $P(\sigma)$ the following

TheOREM 4. If $p=\rho=\tau$ and $\infty>A \geqq B>0$, then $l_{I}<\infty$ and $Q_{I} \geqq B \geqq Q_{I} / l_{I}$.

Proof. Obviously in view of Theorems 2 and 3 we need only to prove that $l_{I}<\infty$. The same arguments used in the proofs of those theorems show that the ordinate $\eta$ of the polygon $\pi$ satisfies $-\log \Gamma[\lambda,(A+\epsilon) p e]=y_{1}<\eta<y_{2}$ $=-\log \Gamma[\lambda,(B-\epsilon) p e]$ for any $\epsilon>0$ arbitrarily small and all large $\lambda$. This implies that the length of each side of $\pi$ is not greater than the segment of the tangent to the graph of $y_{2}$ parallel to that side and bounded by its intersections with $y_{1}[9, \mathrm{pp} .42-46]$. If $\lambda_{0}$ is the abscissa of the contact point, we will prove that the abscissas $\lambda_{0}^{\prime}$ and $\lambda_{0}^{\prime \prime}$ of those intersections satisfy

$$
\beta_{1} \lambda_{0}<\lambda_{0}^{\prime}<\lambda_{0}<\lambda_{0}^{\prime \prime}<\beta_{2} \lambda_{0}, \quad 0<\beta_{1}<1<\beta_{2}<\infty,
$$


where the constants $\beta_{1}$ and $\beta_{2}$ depend only on $\epsilon$. It follows then easily that $l_{I}<\left(\beta_{2} / \beta_{1}\right)+\epsilon^{\prime}<\infty$ for any fixed given values of $\epsilon>0$ and $\epsilon^{\prime}>0$.

To establish (24) we consider the equation of the tangent to $y_{2}$ at the point of abscissa $\lambda_{0}$ :

$$
y=\lambda \lambda_{0} \phi^{\prime}\left(\lambda_{0}\right)+\lambda \phi\left(\lambda_{0}\right)-(\lambda / p) \log [(B-\epsilon) p e]-\lambda_{0}^{2} \phi^{\prime}\left(\lambda_{0}\right) .
$$

The abscissas of the intersections with $y_{1}$ are the roots of the equation $F(\lambda) \equiv\left[\phi(\lambda)-\phi\left(\lambda_{0}\right)\right] \lambda+(\lambda / p) \log [(B-\epsilon) /(A+\epsilon)]-\left(\lambda-\lambda_{0}\right) \lambda_{0} \phi^{\prime}\left(\lambda_{0}\right)=0$. With $\gamma=(B-\epsilon) /(A+\epsilon)$ and $\lambda=\beta \lambda_{0}$ this equation is equivalent to the following equation in $\beta$ :

$$
\begin{aligned}
H\left(\beta, \lambda_{0}\right) \equiv & F\left(\beta \lambda_{0}\right) / \lambda_{0}=\left[\log \left(\beta \lambda_{0}\right) / \varphi\left(\beta \lambda_{0}\right)-\log \lambda_{0} / \varphi\left(\lambda_{0}\right)\right] \beta+(\beta / p) \log \gamma \\
& -(\beta-1)\left[\varphi\left(\lambda_{0}\right)-\lambda_{0} \varphi^{\prime}\left(\lambda_{0}\right) \log \lambda_{0}\right] / \varphi\left(\lambda_{0}\right)^{2}=0 .
\end{aligned}
$$

Now, for any fixed $\beta>0$ we have

$$
\begin{aligned}
\lim _{\lambda_{0}=\infty} & {\left[\log \left(\beta \lambda_{0}\right) / \varphi\left(\beta \lambda_{0}\right)-\log \lambda_{0} / \varphi\left(\lambda_{0}\right)\right] } \\
= & \lim _{\lambda_{0}=\infty}\left\{\log \beta / \varphi\left(\beta \lambda_{0}\right)+\left[1 / \varphi\left(\beta \lambda_{0}\right)-1 / \varphi\left(\lambda_{0}\right)\right] \log \lambda_{0}\right\}=(1 / p) \log \beta
\end{aligned}
$$

because, by the mean value theorem,

$$
\begin{aligned}
& {\left[1 / \varphi\left(\beta \lambda_{0}\right)-1 / \varphi\left(\lambda_{0}\right)\right] \log \lambda_{0}=(\beta-1) \lambda_{0 \varphi^{\prime}}\left(\lambda_{0}^{*}\right) \log \lambda_{0} / \varphi\left(\lambda_{0}^{*}\right)^{2}} \\
& \quad=(\beta-1)\left[\lambda_{0}^{*} \varphi^{\prime}\left(\lambda_{0}^{*}\right) \log \lambda_{0}^{*}\right]\left(\lambda_{0}^{*} / \lambda_{0}^{*}\right)\left(\log \lambda_{0}^{*} / \log \lambda_{0}^{*}\right) / \varphi\left(\lambda_{0}^{*}\right)^{2} \rightarrow 0 \quad \text { for } \lambda_{0}^{*} \rightarrow \infty
\end{aligned}
$$

as $\lambda_{0} \rightarrow \infty$ and the factor in brackets tends to zero as all the others remain bounded, and $\varphi\left(\lambda_{0}^{*}\right)^{2} \rightarrow p^{2}>0$.

We conclude from (25) and (26) that

$$
\lim _{\lambda_{0}=\infty} H\left(\beta, \lambda_{0}\right)=(\beta / p) \log (\beta \gamma)-(\beta-1) / p .
$$

This expression is negative for $\beta=1$, but positive for all $\beta>\beta_{2}$ for some finite large enough $\beta_{2}$, and also positive for all $\beta$ such that $0<\beta<\beta_{1}$ for some finite $\beta_{1},\left(0<\beta_{1}<1\right)$, because the limits of $(27)$ as $\beta \rightarrow \infty$ and $\beta \rightarrow 0$ are respectively $+\infty$ and $1 / p$. It is therefore possible to find a constant $C(\epsilon)$ such that for all $\lambda_{0}>C(\epsilon)$ we have $F\left(\beta_{r} \lambda_{0}\right) \equiv \lambda_{0} H\left(\beta_{r}, \lambda_{0}\right)>0(r=1,2)$ and this together with $F\left(\lambda_{0}\right)<0$ proves both (24) and the theorem.

\section{RefERENCES}

1. A. G. Azpeitia, On the maximum modulus and the maximum term of an entire Dirichlet series, Proc. Amer. Math. Soc. 12 (1961), 717-721.

2. - El orden precisado en las funciones enteras, Rev. Mat. Hisp. Amer. (4) 14 (1954), 3-25, 83-103, 179-193, 221-236.

3. Q. I. Rahman, On the lower order of entire functions defined by Dirichlet series, Quart. J. Math. Oxford Ser. (2) 7 (1956), 96-99. 
4. J. F. Ritt, On certain points in the theory of Dirichlet series, Amer. J. Math. 50 (1928),

5. S. M. Shah, On the proximate orders of integral functions, Bull. Amer. Math. Soc. 52 (1946), 326-328.

6. K. Suguimura, Übertragung einiger Sätze aus der Theorie der ganzen Funktionen auf Dirichletsche Reihen, Math. Z. 29 (1928-29), 264-277.

7. F. Sunyer i Balaguer, Sobre la distribucion de los valores de una funcion entera representada por una serie de Dirichlet lagunar, Rev. Acad. Ci. Zaragoza (2) 5 (1950), 25-73.

8. E. C. Titchmarsh, The theory of functions, 2nd ed., Oxford Univ. Press, London, 1958.

9. G. Valiron, Lectures on the general theory of integral functions, Chelsea, New York, 1949.

UNIVERSITY OF MASSACHUSETTS,

AMHERST, MASSACHUSETTS

BROWN UNIVERSITY,

Providence, Rhode Island 